\title{
Les affects au ceur des préoccupations urbaines et urbanistiques: la réintroduction du sensible pour penser et concevoir la ville
}

\author{
Béatrice Bochet, Lausanne
}

\section{Introduction}

Si la géographie a, entre autres, pour vocation de penser et de rendre compte des relations que les humains et les sociétés entretiennent avec leur territoire, quel est dès lors le poids et le rôle de la dimension socio-affective, longtemps négligée? Il s'agirait ici - en osant paraphraser WUNENBURGER (1997) et sa manière de traiter de la philosophie des images et de la valeur signifiante de l'espace des formes ou des formes dans l'espace - de travailler sur la prégnance cognitive de l'espace, c'està-dire sur la manière dont la ville «prend place dans un vécu, se charge d'affects, inaugure un appel de sens» (Wunenburger 1997: 204-205). Dans cette approche, la ville fait naître quelque chose chez son habitant, fait résonner en lui un phénomène affectif qui est un effet du monde sur sa sensibilité, traduisant sa capacité à activer de la pensée ou, à l'inverse, le pouvoir de la pensée de se reconnaître dans cette ville, de se ressourcer par le biais d'un rapport affectif, émotionnel à son espace. Il s'agit de mener une réflexion sur la constitution préalable des liens entre les sujets et leurs espaces urbains, plus précisément sur les affects et les émotions. Ce questionnement est nécessaire à la compréhension du désir d'habiter l'urbain, de le fuir (étalement urbain), de l'éviter (par multiplication des gated communities), voire de comprendre son renouveau actuel, tel que l'exprime l'expression devenue courante dans la littérature: «back to the city». Cette expression géographique de nouvelles logiques pro-urbaines apparemment émergentes valide in fine le postulat voulant que la ville peut être aimée et qu'elle est en train de redevenir attirante en dépit de la forte prégnance des idéologies anti-urbaines (Bochet 2000, 2007).

Cette lecture peut se faire à travers l'écologie de la perception développée par JAMES GIBSON, rappelant que les orientations perceptives du sujet sont indissociables des «prises» ou «offrandes» du site. Le terme de «prise» a été proposé par BERQUE comme traduction de l'«affordance» (Aufforderung en allemand). Citant Gibson, Berque (1990: 102) affirme que les prises correspondent à «ce qu'un environnement spécifique fournit (affords) à un observateur qui peut (affords) le percevoir parce que lui-même est spécifiquement adapté à cet environnement. (...) Les affordances sont des propriétés (de l'objet) prises en référence à l'observateur. Elles ne sont ni physiques, ni phénoménales».
Les prises sont des propriétés invariantes de l'objet, comme dirait Gibson, bien qu'elles n'existent en tant que prises que dans et par une certaine relation.

C'est en se référant à cette notion d'affordance (GiBson 1977) et à celle d'habitus (Bourdieu 1980) que BERQUE affirme que la réalité ne peut être considérée comme seulement «produite» ou «construite»par la société du point de vue de la médiance (comme elle était définie en 1990), car elle ne se manifeste que par trajection entre les deux pôles théoriques du sujet (les acteurs sociaux) et de l'objet (les choses) ou encore de l'actif et du passif. Du point de vue de la médiance, la notion de prise s'élargit par elle-même:

«un milieu se manifeste en effet comme un ensemble de prise avec lequel nous sommes en prise; des ressources et des contraintes, des risques et des agréments que la réalité comporte dans la mesure où elle nous comporte aussi» (Berque 1990: 100).

Cela signifie que la ville offre une prise à un phénomène qui sera qualifié ici d'affectif. Ces prises vont permettre au sujet de s'approprier ou de rejeter un espace, de se ressourcer par le biais d'un rapport affectif. À la même idée (de prise) se rattachent le mot «emprise» et son contraire, «déprise», qui correspond à l'abandon et au rétrécissement du territoire. L'espace offre alors peut-être des prises à la «dé»représentation, à la «dé»perception et à la «dé»action: certaines villes, certains quartiers se donnent à la représentation, d'autres se refusent. Dans cette optique, la perception se déploie moins dans un milieu qu'en fonction d'un milieu. Elle doit donc être rapportée aux qualités du site étudié, tout comme aux qualités des acteurs sociaux. Ce questionnement révèle un nouvel objet du savoir: les affects urbains, c'est-à-dire le rapport affectif à la ville, et implique la mobilisation d'un nouveau référentiel en termes d'analyse urbaine: le socio-affectif.

Ainsi, les affects semblent devoir intéresser au premier chef les géographes, au vu des enjeux théoriques, méthodologiques et/ou pragmatiques de leur discipline. Au niveau théorique, l'enjeu est de taille; il peut être défini comme une tentative de resituer la question des affects et du rapport affectif à la ville dans le débat sur l'urbain et ainsi d'échapper à certains dogmes bien établis qui «interdisent» la prise en compte des affects dans l'analyse urbaine. L'enjeu pragmatique principal peut être défini quant à lui comme une résistance aux tendances évolutives dominantes en favorisant l'introduction de cette notion de rapport affectif dans la 


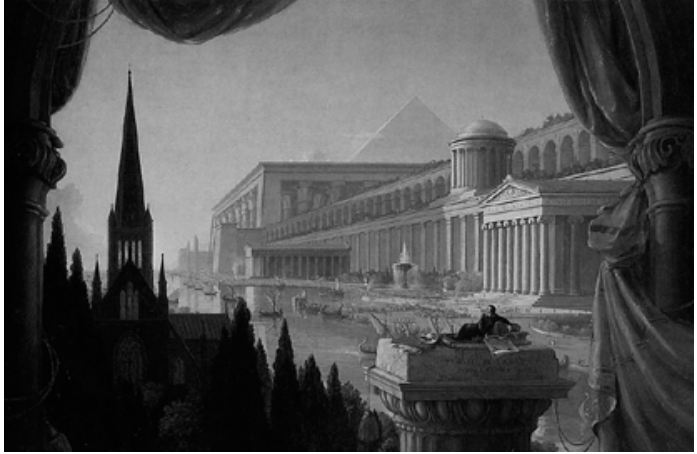

Photo 1: Thomas Cole: The Architect's Dream, 1840 Thomas Cole: Der Traum des Architekten, 1840

Thomas Cole: La rêve de l'architecte, 1840

Source: http://upload.wikimedia.org/wikipedia/commons/ c/c6/Cole_Thomas_The_Architect-s_Dream_1840.jpg

pratique de l'urbanisme opérationnel ordinaire. En effet, il est manifeste que, derrière les enjeux méthodologiques, se cache une volonté d'invention de principes inédits ou de techniques d'évaluation nouvelles, pour tenter de réintroduire l'affect dans la situation de l'interview et générer un processus permettant de voir celui-ci évoluer. Le propos vise ici à faire état de ces enjeux et des perspectives de recherche s'y rapportant.

\section{Vers une anthropologie du rapport affectif?}

D'un point de vue strictement théorique, ce questionnement exploratoire est conçu comme une contribution à la constitution d'un nouveau champ du savoir: une anthropologie du rapport affectif. Schématiquement, l'étude du rapport affectif a donné lieu jusqu'à présent à deux types d'approches.

La première approche trouve ses origines dans le développement de la géographie sociale des années 1940 aux États-Unis et des années 1960 en France. À partir de cette époque, les chercheurs s'intéressent pleinement à la diversité psychologique et sociale des relations de l'homme à l'espace, puis au processus des représentations (LÉVy-LEBOYER 1980; MOLES \& RoHMER 1978). Cette perspective de recherche est définitivement lancée et adoptée au début des années 1970 en France. De LYNCH (1960) à LEDRUT (1973), la recherche a progressivement permis d'identifier, en termes complémentaires, l'identité, la structure et les significations pouvant correspondre à la relation pratique et émotive du sujet à l'objet urbain et le rôle des images comme expression partielle du contenu subjectif et affectif de la ville, structure vivante de rencontres, de conflits et de créations. En effet, la ville est souvent rêvée avant même le discours sur elle, et bien sûr avant sa réalisation (Photo 1). Ne dit-on pas d'une cité qu'elle est triste, gaie, grisée, ensoleillée, dynamique ou conservatrice? Tant la littérature que les travaux scientifiques sont remplis de ce type de notations. Il est de fait que pour chacun de nous existent des lieux de pouvoir, de liberté, de plaisir, renvoyant aux notions chères à Y.-F. TuAN (1974) de «topophilie» et de «géopiété». La première concerne la description des lieux que les gens affectionnent et auxquels ils se sentent attachés, alors que la seconde exprime le caractère mythique et religieux des lieux. Certains auteurs vont jusqu'à parler de «génie des lieux» lorsque ceux-ci, par «leur grandeur», mobilisent l'imaginaire humain. La topophobie est évoquée pour les lieux qui font peur, sentiments ressentis par plus de personnes qu'on ne le croit en général précisent BAILLY \& Pocock (1995). Bachelard (1957) parle également de topophilie pour les lieux aimés et de topophobie pour les lieux de terreur. De son côté, s'agissant des distances, FrÉmont (1976) évoque la distance affective, celle qui intègre le rapport sensible aux lieux, qu'il faut distinguer de la distance standard, de celle liée à la géométrie, de la distance structurale et de celle identifiant des relations économiques et sociales. Dès lors, la «notion» de représentation s'avère indispensable. C'est le début de l'analyse des représentations mentales. Ces analyses introduisent en quelque sorte la première prise en compte d'un processus subjectif et affectif.

Plus tardivement, d'autres travaux ont été menés sur la recherche du bien-être, cette problématique étant assimilée à la quête d'une vie meilleure et d'un bienêtre social et spatial, parallèlement à l'émergence des préoccupations du développement urbain durable. Les premières recherches menées en Amérique du Nord sur la recherche du bien-être montrent le rôle des éléments économiques (situation financière) et environnementaux (habitats, loisirs), mais aussi le rôle des éléments personnels (amour, famille) pour saisir le pourquoi des jugements. L'aspect spatial, indispensable composante du vécu humain, fait aussi partie intégrante des éléments contribuant au bien-être, ce qui confirme l'importance de l'espace vécu. Ce sont ainsi des situations très personnelles qui marquent la satisfaction des individus, même si celles-ci sont nécessairement intégrées dans le tissu des relations sociales. On note que si l'intérêt pour l'étude des représentations a été motivé par le souci de mieux comprendre le «moteur des actions humaines», malgré l'assimilation de paramètres économiques de plus en plus nombreux, les indicateurs matériels ne reflètent pas nécessairement la satisfaction de la population et n'intègrent ni l'espace social, ni l'espace vécu (BAILlY 1981; RACINE 1985 a et b). Par suite de l'inexorable décalage entre 
aspirations et représentations, le bien-être ne se réduit jamais aux seuls critères économiques.

Indépendamment mais curieusement en parallèle de ce qui se passait dans la géographie anglo-saxonne, c'est après en avoir posé le principe à l'échelle de la ville suite aux travaux de MARTOUZET sur Fort-deFrance (2002) (Bochet 2000; Bochet \& RaCine 2002) qu'on a découvert que ce thème des affects commençait à être discuté par toute une série d'auteurs anglais et américains. Ce thème a d'ailleurs fait l'objet d'une session organisée par Dewsbury et Lea de l'Université de Bristol au congrès annuel de l'Association of American Geographers à Denver (Colorado) en 2005. Les travaux ont alors été principalement orientés par la question de savoir dans quelle mesure la géographie pouvait être enrichie, voire renouvelée par la prise en compte du fait que le monde humain était construit et vécu à travers les émotions/les affects. Ces travaux ont considéré que les relations sociales sont vécues à travers les émotions, même si jusqu'ici force était de constater que les qualités émotionnelles de la vie sociale étaient rarement rendues apparentes au sein du lexique de la recherche sociale.

Il demeure que l'émergence du paradigme émotionnel est aujourd'hui avérée, en Amérique du Nord (ANDERson 2006; Davidson, Bondi \& SMITH 2007; ThiEn 2005) comme en Europe (RIMÉ 2005). On n'est donc pas ou plus seul à penser que cette omission du thème des affects et des émotions se traduit par une compréhension incomplète de la marche du monde: négliger les composantes émotionnelles de la relation au monde revient à exclure un ensemble clé de relations à travers lesquelles les sociétés sont faites et vécues (ANDERSON $\&$ SMITH 2001). D'où cette tentative qui vise à investir un champ encore très largement inexploré sur la base d'interrogations jugées vitales et terriblement actuelles pour le devenir des cités, au double plan des connaissances fondamentales et de l'action.

\section{Exploration d'un modèle des prises affectives à l'es- pace urbain à travers urbanité, aménités et civilité}

D'un point de vue pragmatique, l'enjeu est de faire évoluer la pratique de l'urbanisme opérationnel ordinaire en intégrant cette dimension des affects. Sans prétendre que le défi sera facile à résoudre, il paraît que la caractérisation et la détermination des rapports affectifs et émotionnels à la ville et de leur rôle éventuel vis-à-vis du projet urbain deviennent centrales dans l'explicitation d'un rapport à une urbanité désirée. Certes, des rapports affectifs à la ville ne peuvent guider à eux seuls un urbanisme ou une politique urbaine; cependant, il devient essentiel de prendre en compte que la relation à la ville semble comporter de fortes composantes affectives et que celles-ci sont en rapport avec différents systèmes de valorisation, d'autant que la première qualité d'un aménagement urbain semble devoir rendre cette relation la plus positive possible. Ce travail veut donc rendre possible la reconnaissance d'éléments favorisant la valorisation d'un rapport affectif positif à la ville afin d'en tenir compte sur le plan pratique et politique pour la pratique de l'aménagement, conçue en termes de projet urbain négocié et distribué (CALlon 1997), susceptible d'être approuvé.

Au lieu d'aborder le ou les rapports à la ville par la négative en recherchant les conditions de rejet d'un lieu (dysfonctionnements affectifs), on l'abordera par la positive en recherchant prioritairement les conditions d'acceptabilité d'un lieu, même si l'étude des facteurs de l'un va entraîner indirectement une meilleure connaissance de l'autre. On cherche donc non seulement à comprendre et à lutter contre les dysfonctionnements affectifs, mais aussi à comprendre et à rechercher ce qui fait qu'un lieu devient acceptable pour la majeure partie de la population résidente. Si un travail sur l'ensemble des déterminants potentiels de l'affect, concept lui-même extraordinairement complexe selon ANSART (1999), ne semble pas réaliste, les hypothèses invitent à penser qu'une analyse peut être conduite à travers les qualités du site étudié et celles des acteurs-observateurs (Bochet 2000). Pour cela, on part, comme mentionné dans l'introduction, d'un modèle développé à partir de la notion d'«affordance» de GiBson, traduite sous le terme de «prise» par Berque. Ces prises se déploient moins dans un milieu qu'en fonction d'un milieu, et renvoient de fait autant aux qualités du site étudié qu'aux qualités des acteurs sociaux, les deux dimensions jugées hypothétiques du rapport à la ville.

D'une part, il s'agit de considérer les dimensions du sujet, c'est-à-dire quelques-unes au moins des dimensions individuelles, sociales et culturelles considérées par hypothèse comme facteurs potentiels de la plus ou moins grande affectivité qu'un individu porte à la ville selon son âge, son expérience et le tissu relationnel et social dans lequel il est inscrit. D'autre part, il s'agit de prendre en considération les caractéristiques de l'objet (la ville) à travers les dimensions spatiale, sociale et sociopolitique qu'on caractérise par les aménités, l'urbanité et la civilité.

- Les aménités désignent le contexte matériel et physique/spatial local, les facilités offertes par la ville et ce qu'elles permettent, l'ensemble des aspects concrets et matériels de la ville et l'offre urbaine qui en résulte. Il s'agit alors des avantages et désavantages procurés par la ville au plan fonctionnel, communicationnel mais aussi esthétique. 
- L'urbanité est définie par la plupart des géographes comme la qualité d'une organisation urbaine illustrant l'identité d'une ville, sa mémoire, ses conflits, ses changements, certains auteurs se focalisant plutôt sur la qualité d'un espace ou d'une architecture exprimant et laissant s'exprimer les projets et les comportements des différents acteurs sociaux, d'autres évoquant plutôt des pratiques sociales agissant sur l'espace et l'architecture. L'urbanité renvoie à une dimension sociale dominante et désigne une situation circonstancielle d'interaction liée à une certaine ambiance urbaine. L'urbanité renvoie donc à l'ensemble des relations interpersonnelles qui existent ou se créent dans la ville par l'intermédiaire de l'art de vivre spécifique aux villes. L'amabilité, la sociabilité, la politesse, la convivialité, la tolérance, la liberté, l'intégration, la sympathie mais aussi l'indifférenciation, l'anonymat, l'exclusion, la solitude, l'isolement sont des valeurs sous-jacentes à la notion d'urbanité. Ces valeurs renvoient aussi à une grande diversité de représentations en ce qui concerne la qualité de vie urbaine (p.ex. la tolérance, la mixité, la diversité) (Bochet 2000) et peut être alors tout simplement à ce que LeDrut (1973) appelle les valeurs humaines que font intervenir le milieu social et son esprit: soit l'ambiance sociale et la mentalité des habitants.

- Quant à la civilité, dimension plus sociopolitique, elle se définit par la place occupée par un individu dans le groupe et par un ensemble de pratiques collectives et de codes de conduites partagées servant de support aux liens sociaux et permettant à chacun de trouver une place dans la société et de restaurer un sentiment d'appartenance à une même collectivité citadine.

Ces trois dimensions, spatiale, sociale et sociopolitique «conditionnent» trois types de prises à la ville. Par l'intermédiaire des aménités, le sujet dans ce contexte entretenant des relations utilitaires, fonctionnelles avec la ville par le biais des parkings, centres commerciaux, bancs, bureaux, infrastructures sportives et culturelles, feux rouges, parcs urbains. Ce premier type de rapport à la ville, directement lié aux aménités, peut être identifié comme fonctionnel. A travers l'urbanité, le sujet vit plus sa relation avec le milieu qu'il ressent (anonymat, hétérogénéité, contact, harmonie, cosmopolitisme, réseau, solitude, foule, chaos intensité). Il ne s'agit plus simplement d'une relation utilitaire avec la ville, mais d'une situation vécue dans un contexte urbain, ce rapport pouvant être identifié comme plus relationnel avec la ville. A travers la civilité, dimension plus sociopolitique, la relation n'est plus du tout utilitaire ni tout à fait relationnelle (communauté, pouvoir, sécurité, violence, politique, appartenance, citoyen, vie sociale). Il peut alors s'agir d'une relation plus perceptive au milieu urbain.
Ces trois dimensions deviennent des prises avec lesquelles les acteurs sont en prise, c'est-à-dire que cellesci vont varier en fonction des qualités des acteurs. BERQUe (1990: 101) explique cela en affirmant que «pour réelles que soient les prises médiales, elles ne le sont jamais pour tout le monde, car elles ne possèdent pas l'universalité de l'objet physique. Dire qu'elles se réalisent dans une relation dont l'un des termes est la société, c'est sous-entendre que celle-ci leur accordera plus ou moins de valeur suivant ses propres déterminations; lesquelles sont des rapports sociaux. Les prises n'existent donc que dans certains milieux, de manière plus ou moins consistante, collective, précoce ou tardive suivant les acteurs sociaux considérés».

Qu'en est-il dès lors des trois qualités du site que l'on mettrait en rapport avec les qualités des acteurs? Le croisement des dimensions du sujet (facteurs personnels, sociaux et culturels) et les caractéristiques de la ville (aménités, urbanité et civilité) permettent de définir une typologie exploratoire des rapports affectifs à l'espace. Cette typologie peut être caractérisée selon différents critères d'analyse tels que le ou les type(s) et variété(s) des rapports, l'intensité qui décrit le rapport faible ou fort à la ville, la conscience du rapport subi ou désiré, la variation temporelle (Tableau 1), mais aussi par rapport à des façons de qualifier le monde. Sur ce point, considère-t-on le rapport affectif selon «le milieu/environnement/paysage» de BERQUe ou fait-on une analogie avec les trois écoutes du monde d'Amphoux et al. (2001) (Tableau 2)? Faut-il parler d'un environnement affectif, extérieur au sujet mais avec lequel celui-ci entretient des relations fonctionnelles, d'un milieu affectif dans lequel le sujet est plongé et avec lequel il entretient des relations à travers ses activités, ou d'un paysage affectif, intérieur et extérieur à la fois, avec lequel le sujet entretient des relations perceptives?

\section{Test méthodologique ou comment réintroduire l'affect dans la saisie du rapport à l'espace?}

L'objet de la recherche consiste tout d'abord, on vient de le voir, à mettre au point un outil d'analyse des rapports d'affects au milieu urbain à différentes échelles. Dans un deuxième temps, pour tester cet outil d'analyse, l'enjeu méthodologique consistera, comme déjà dit, à expliciter de manière réflexive et critique la manière de réintroduire l'affect dans la situation de l'interview.

Tout en en reconnaissant les risques, il faut préciser que ce questionnement n'est pas forcément l'expression d'un retour aux beaux jours de l'idiographisme ou d'une préoccupation pour l'unicité des êtres et des lieux qu'on aurait coloré des légitimations de la postmodernité. On prétend que ce questionnement peut 


\begin{tabular}{|c|c|c|c|}
\hline $\begin{array}{c}\text { Éléments } \begin{array}{c}\text { Éléments } \\
\text { urbains }\end{array} \\
\text { Facteurs personnels }\end{array}$ & Aménités & Urbanité & Civilité \\
\hline Facteurs sociaux & $\begin{array}{c}\text { Fonctionnel } \\
\text { Relationnel }\end{array}$ & $\begin{array}{c}\text { Relationnel } \\
\text { Fonctionnel }\end{array}$ & $\begin{array}{c}\text { Perceptif } \\
\text { Fonctionnel }\end{array}$ \\
\hline Facteurs culturels & $\begin{array}{c}\text { Fonctionnel } \\
\text { Perceptif }\end{array}$ & $\begin{array}{c}\text { Relationnel } \\
\text { Perceptif }\end{array}$ & $\begin{array}{c}\text { Perceptif } \\
\text { Relationnel }\end{array}$ \\
\hline
\end{tabular}

Tab. 1: Typologie exploratoire des rapports affectifs à l'espace

Explorative Typologie der affektiven Beziehungen zum Raum

Exploratory typology of affective relations to space

continuer à s'inscrire dans des préoccupations authentiquement nomothétiques, à la recherche des éléments communs et généraux sous-tendant les sources de variation des rapports des habitants à la ville (DAviES 1995; Davies \& Herbert 1993; Davies, Ki Chan \& TOWNSHEND 1999). Si

«remonter de l'observation à l'imaginaire affectif paraît un problème d'ordre individuel, une telle étude pourrait cependant révéler quelque chose de pertinent aussi du point de vue social et en particulier de la socialité»

écrivait Noschis (1984: 46) dans son ouvrage sur la Signification affective du quartier. Et le même auteur de préciser que

«la typification de l'expérience individuelle est un procédé de généralisation et permet, dans le cas d'une collectivité d'explorer des régularités dans l'interaction quotidienne des gens».

\subsection{Une approche multiple et cumulative}

Compte tenu de la forte charge affective qui entoure cette problématique, on part néanmoins du principe qu'en regard des difficultés reconnues d'accès des affects à la conscience réflexive, celle-ci ne peut être qu'indirecte, «multiple et cumulative» et reposer sur le principe de «récurrence». Pour ce faire, on s'appuie sur les travaux d'AMpHoux (2001:152) qui indique que pour

«limiter l'arbitraire de l'interprétation et parvenir à objectiver des phénomènes, il faut croiser des interprétations différentes, et contrôler de la sorte le taux d'objectivité de ce qu'on avance en fonction d'un taux de redondance intersubjective».

L'intérêt de cette approche est de multiplier les démarches afin de constituer des corpus différents sur le même thème. La méthode d'investigation va mettre en jeu plusieurs situations d'enquêtes, renvoyant à diffé- rents contextes de production de données empiriques, à partir desquels vont se constituer plusieurs types de corpus. En reposant sur un «artifice de représentation» (le dessin de cartes mentales, la réunion de groupe ou la présentation d'images photographiques), ces méthodes consistent à libérer la parole de la personne interviewée. Dans tous les cas, Amphoux dit (2001), qu'on recueille des discours riches et foisonnants, qui sont certes plus hésitants que si le questionnement était direct, mais qui, du coup, révèlent en négatif les fondements d'une pratique réelle de l'espace et d'un rapport sensible à la ville.

\subsection{Un procédé d'écriture}

De ces matériaux qui livrent un discours par «émergence, instable et toujours renégociable» (Амpноux 2001:154), il s'agit alors de préciser des règles de recomposition. On peut déjà se focaliser dans un premier temps sur le repérage des contradictions (ce qui suppose un système logique d'évaluation monovalente), plutôt que sur le repérage de résonances, convergences et homologies, ce qui suppose un art de construire, de repérer et de recomposer le sens commun de données hétérogènes. Dans cette démarche, il est probable que l'on puisse découvrir bien des contradictions, des erreurs de jugements et des représentations ou désirs incohérents, tels que le fait par exemple de vouloir à la fois de l'espace, des forêts intactes, du travail, une voiture, des moyens de communication et des grandes surfaces dans le même secteur. Ces formes d'incohérences, ces «erreurs» par rapport à la réalité qui n'en sont pas en fait, peuvent signifier beaucoup de choses et renvoyer à des thématiques tout à fait différentes: capacité d'adaptabilité à un milieu, malaise urbain, définition de besoins à satisfaire, expression des relations entre représentations et comportements, modèles d'attitudes tels que le NIMBY («Not In My Back- 


\begin{tabular}{|l|l|l|}
\hline Environnement affectif & Milieu affectif & Paysage affectif \\
\hline Démarches in situ & Démarches off situ & Démarches out situ \\
- Rapport fonctionnel & - Rapport relationnel & - Rapport perceptif \\
- Aménités & - Urbanité & - Civilité \\
- Spatial & - Social & - Sociopolitique \\
- Connu & - Vécu & - Perçu \\
\hline
\end{tabular}

Tab. 2: Modèle exploratoire provisoire

Provisorisches exploratives Modell

Provisional exploratory model

yard»). La question ici est de trouver à quel niveau de généralisation, de conceptualisation, de théorisation plus ou moins inconsciente de la part de l'habitant, il n'existe plus de contradiction. Il n'est d'ailleurs pas sûr qu'il existe un niveau suffisamment abstrait qui puisse résoudre toutes les contradictions.

\subsection{Un échantillon expressif et trois types de rapport à l'image et à la ville}

Dans l'optique de l'écologie de la perception et des prises de Gibson, Thibaud (2001: 83) constate

«qu'il n'y a de prises au monde que dans une situation d'interaction avec le monde, que quand il y a mouvement.

Il serait illusoire de vouloir dissocier la perception du mouvement».

Admettre cette emprise du

«contexte environnemental de la perception conduit à développer des techniques d'investigation in situ. Par ailleurs, rendre compte de l'activité perceptive des citoyens suppose d'approcher au plus près leur propre point de vue. Les façons de percevoir sont donc indissociables du cours d'action dans lequel le passant est engagé» (ibid.).

Ce principe devient opérant pour saisir la construction sensorielle de l'espace. Le «bougé» se donne comme fondement de la perception. La multiplication des démarches s'associe à une diversité d'angles d'approches des différents phénomènes qu'on souhaite étudier selon le modèle cinématographique, avec une caméra se déplaçant selon différents angles de vues, mais toujours in situ. A des enquêtes effectuées sur les lieux se superposent nécessairement des enquêtes dites out situ: il s'agira toujours de traiter d'un lieu, mais depuis un autre endroit, à des enquêtes dites off situ, littéralement «au-dessus du lieu», c'est-à-dire que la caméra est au-dessus de ce que l'on étudie, imperceptible. In situ, out situ et off situ désignent trois types de rapports à l'image et à la ville. Faut-il alors rapprocher les déterminants et les angles d'approche? Les aménités en approche in situ, pour se démarquer des démarches expérimentales extraites du contexte? L'urbanité à travers des enquêtes off situ, car elle dépasse le contexte physi- que local? Quant à la civilité, doit-elle être abordée selon une démarche en out situ?

\section{Conclusion}

Les divers enjeux évoqués dans cet article souhaitent contribuer à donner plus de pertinence sociale et politique à la géographie. Il s'agit en particulier de faire émerger ce qui restait de côté, de lutter contre cet effacement d'une dimension essentielle de l'expérience humaine que sont les affects et leurs soubassements psychanalytiques des relations aux lieux, et d'investir le champ des affects et des émotions qui font que le monde qu'on connaît reste encore un domaine spectaculairement inexploré, inconnu dans bien des choses qu'on fait et, pire encore, dans virtuellement toutes les politiques qu'on pourrait recommander. S'agit-il d'une question relevant de l'air du temps? Pour certains sans doute, comme le relève LUNGINBÜHL (2006: 128):

«devant la fin théorique des idéologies, ou plus exactement devant la suprématie de l'idéologie néo-libérale, il semble qu'une autre idéologie est née, qui l'accompagne, celle que je nommerai 〈l'idéologie de l'émotionnel〉 assez propre à l'activité du paysagisme et à l'essor du marché».

Il s'agit sans doute également d'une réponse à un vrai besoin. Sur le plan social, qu'en est-il de la capacité ou incapacité à aimer la ville, à comprendre en quoi et pourquoi on l'aime, et plus généralement, quels sont les déterminants du rapport affectif à la ville? Sur le plan strictement territorial également, qu'en est-il de l'incapacité à construire un modèle urbain réellement satisfaisant au double sens de la forme et des choix de ses acteurs? Ce modèle pourra-t-il rendre compte en particulier de la possibilité qu'ont les habitants d'aimer leur ville et de choisir d'y résider? On est bien là dans le registre des affects et des émotions. Ce champ d'analyse exploratoire prend aujourd'hui tout son sens au vu de la réflexion sur la ville insoutenable et les conditions de l'habitabilité sur la terre (BERQUE et al. 2006). 


\section{Bibliographie}

Amphoux, P. (2001): L'observation récurrente. - In: Grosjean, M. \& J.-P. Thibaud (dirs): L'espace urbain en méthodes. - Marseille: Parenthèses: 153-173.

Amphoux, P., avec Jaccoud, C., Meier, H., Meier-DalLACH, H.-P., Gehring, M., Bardyn, J.-L. \& G. ChelKOFF (coll.) (2001): Aux écoutes de la ville. La qualité sonore des espaces publics européens. Méthode d'analyse comparative. Enquête sur trois villes suisses. Lausanne: Institut de Recherche sur l'Environnement Construit (IREC), rapport $\mathrm{n}^{\circ} 94$.

Anderson, B. (2006): Becoming and being hopeful: towards a theory of affect. - In: Environment and Planning D: Society and Space 24: 733-752.

Anderson, K. \& S.J. Smith (2001): Emotional geographies. - In: Transactions of the Institute of British Geographers 26: 7-10.

Ansart, P. (1999): Affects ou affectivité. - In: AnsART, P. \& A. Akoun (dirs): Dictionnaire de sociologie. - Paris: Seuil.

Bachelard, G. (1957): La poétique de l'espace. - Paris: Presses Universitaires de France.

Bailly, A. (1981): Géographie du bien-être. - Paris: Presses Universitaires de France.

Bailly, A. \& D. Pocock (1995): L'humanisme en géographie. - In: BAILLY, A. (coord.): Les concepts de la géographie humaine. - Paris: Masson: 165-171.

Berque, A. (1990): Médiance. De milieux en paysages.

- Montpellier: GIP Reclus.

Berque, A. Bonnin, P. \& C. Ghorra-Gobin (2006): La ville insoutenable. - Paris: Belin.

BocHet, B. (2000): Le rapport affectif à la ville, essai de méthodologie en vue de rechercher les déterminants du rapport affectif à la ville. - Diplôme d'études approfondies (DEA) de Sciences de la Ville, Maison des sciences de la ville et École d'Ingénieurs Polytechnique - Polytech'Tours - section Aménagement, Université François Rabelais de Tours.

Bochet, B. (2007): La ville comme lieu d'investissement affectif. - In: Actes du colloque «La ville mal aimée, ville à aimer». - Centre Culturel International de Cerisy-la-Salle, 5-12 juin.

Bochet, B. \& J.-B. Racine (2002): Connaître et penser la ville: plaidoyer pour l'exploration des affects et des émotions dans la géographie urbaine. - In: Géocarrefour 4: 117-132.

Bourdieu, P. (1980): Le sens pratique. - Paris: Éditions de Minuit.

Callon, M. (1997): Concevoir: modèle hiérarchique et modèle négocié. - In: M. BonNET (éd.): L'élaboration des projets architecturaux et urbains en Europe. Vol. 1: Les acteurs du projet architectural et urbain. - Paris: Plan, urbanisme, construction, architecture (PUCA).

Davidson, J., Bondi, L. \& M. Smith (éds) (2007): Emotional Geographies. - In: Annals of the Association of American Geographers 97, 2: 448-451.
DAVIES, W.K.D. (1995): The power of communities. - In: Acta Wasaensis 45, 6: 49-75.

Davies, W.K.D. \& D.T. Herbert (1993): Communities within cities. An urban social geography. - Londres: Belhaven.

Davies, W.K.D., Ki Chan, W.J. \& I. Townshend (1999): How do communities differ? Empirical evidence for behavioural and cognitive-affective dimensions of community. - In: Aguilar, A.G. \& I. Escamilla (éds): Problems of megacities, social inequalities, environmental risk and urban governance. - Mexico: Universitad Nacional Autónoma de México: 529-542.

Frémont, A. (1976): La région, espace vécu. - Paris: Flammarion.

Gibson, J.J. (1977): The theory of affordances. - In: Shaw, R. \& J. Bransford (eds): Perceiving, acting and knowing. - Hillsdale, N.J.: Erlbaum.

Ledrut, R (1973): Les images de la ville. - Paris: Anthropos.

Lévy-Leboyer, C. (1980): Psychologie et environnement. - Paris: Presses Universitaires de France.

LungInbüHL, Y. (2006): Habiter la nature: le jardin comme recours, confrontation d'idéologies politiques, économiques et d'idéologies paysagistes. - In: BERQUE, A., Bonnin, P. \& C. Ghorra-Gobin (éds): La ville insoutenable. - Paris: Belin: 118-129.

LyNCH, K. (1960): The image of the city. - Cambridge: The MIT Press.

Martouzet, D. (2002): Le rapport affectif à la ville, conséquences urbaines et spatiales. Le cas de Fort-deFrance. - In: Annales de géographie 111, 623: 73-85.

Moles, A. \& E. Rohmer (1978): Psychologie de l'espace. - Paris: Casterman.

Noschis, K. (1984): Signification affective du quartier. - Paris: Librairie des Méridiens.

RACINE, J.-B. (1985a): La planification de la qualité de la vie: besoin, mythe ou mode? - In: Le phénomène de la mode, cours général public 1984-1985. - Publications de l'Université de Lausanne, fasc. LXV, Lausanne: Payot: 119-140.

RACINE,J.-B. (1985b): Qualità della vita, benessere e mutamento sociale: verso una nuova geografia degli spazi vissuti e dei rapporti dell'uome con il territorio. - In: COPETA, C. (éd.): Esistere ed abitare, prospettive umanistiche nella geografia francophona. - Milan: Angeli: 199-246.

Rimé, B. (2005): Le partage social des émotions. - Paris: Presses Universitaires de France.

THIBAUd, J.-P. (2001): La méthode des parcours commentés. - In: Grosjean, M. \& J.-P. Thibaud (dirs): L'espace urbain en méthodes. - Marseille: Parenthèses: 79-101.

Thien, D. (2005): After or beyond feeling? A consideration of affect and emotion in geography. - In: Area 37, 4: 450-454.

TuAn, Y.-F. (1974): Topophilia. - Englewood Ciffs: Prentice-Hall.

Wunenburger, J.-J. (1997): Philosophie des images. - Paris: Presses Universitaires de France. 


\section{Résumé: Les affects au cour des préoccupations urbaines et urbanistiques: la réintroduction du sensible pour penser et concevoir la ville}

Les affects: un nouvel objet du savoir? Les affects urbains (et leur corollaire le rapport affectif à la ville) mobilisent, à côté des dimensions morphologiques, socio-démographiques et structuro-fonctionnelles, un nouveau référentiel en termes d'analyse urbaine: le registre socio-affectif. Le propos vise ici à faire état de ce courant émergent et des perspectives de recherche s'y rapportant, à travers une explicitation des différents enjeux théoriques, pragmatiques et méthodologiques soulevés par la mobilisation de ce référentiel. Transversalement, il s'agira premièrement de proposer un modèle hypothétique de lecture des prises affectives potentielles offertes aux citadins pour tenter de modéliser conceptuellement la constitution des rapports affectifs des individus envers la ville. Deuxièmement, il s'agira de comprendre les relations parfois antagonistes que les citadins entretiennent avec leurs villes de manière à savoir si ce rapport peut être un moyen d'action susceptible d'améliorer ou de modifier la relation individu/ville et à terme les pratiques urbanistiques.

Mots-clés: rapport affectif à la ville, effet, urbanité, qualité de vie

\section{Zusammenfassung: Affekte als Fokus der Stadtfor- schung: zur Wiedereinführung der Gefühlsebene in der Konzeptualisierung von Stadt}

Die Problematik der Affektivität: ein neuer Wissensgegenstand? Urbane Affekte (und deren Folge, der affektive Bezug zur Stadt) fördern neben den morphologischen, soziodemographischen und strukturfunktionellen Dimensionen ein neues Bezugssystem der urbanen Analyse: das sozio-affektive Spektrum. Der Beitrag stellt diese aufkommende Strömung und die sich daraus ergebenden Forschungsperspektiven dar, indem verschiedene theoretische, pragmatische und methodologische Herausforderungen, die aus diesem Bezugssystem entstehen, spezifiziert werden. Zudem geht es auch darum, ein hypothetisches Modell der Lesart der potentiellen affektiven Wirkung auf die Stadtbewohner vorzustellen. Damit wird versucht, vor dem Experimentieren und der Bildung von affektiven Beziehungen von Individuen der Stadt gegenüber, den manchmal antagonistischen Bezug, welchen die Stadtbewohner mit ihren Städten unterhalten, zu verstehen.
So soll herausgefunden werden, ob dieser Bezug ein Handlungsfeld sein kann, das erlaubt, die Beziehung Individuum/Stadt und damit auch die Stadtenwicklung zu verbessern und zu verändern.

Schlüsselwörter: affektiver Bezug zur Stadt, Wirkung, Urbanität, Lebensqualität

\section{Summary: Affectivity as urban research focus: reintroduction of personal feelings in urban concep- tualization}

The article explores the status of affectivity within the field of urban geography. It is argued that urban affectivity (and as a consequence, the affective relation to a city) encourages a new relational system in urban analysis - the socio-affective sphere - to compliment the existing morphological, socio-demographic and structural-functional dimensions. This upcoming research paradigm and related research perspectives are described from the point of view of the theoretical, pragmatic and methodological challenges faced. Furthermore, a hypothetic model is presented of interpretations regarding potential affective effect on urban dwellers. This should allow a greater understanding of the, at times, antagonistic attitude individuals show towards a city before a step is taken towards experimentation and development of individual affective urban space relations. It is hoped that the research of this early relation will help define whether or not it may be seen as a field of action that allows not only a better understanding of the relation between individuals and urban spaces but also its development.

Keywords: affective relation to urban space, effect, urbanity, quality of life

Dr. Béatrice Bochet, Observatoire de la ville et du développement durable, Institut de géographie, Université de Lausanne, Bâtiment Anthropôle, Dorigny, CH-1015 Lausanne, Suisse.

e-mail: Beatrice.Bochet@unil.ch

\section{Manuskripteingang/received/manuscrit entré le} 8.6.2008

Annahme zum Druck/accepted for publication/accepté pour l'impression: 24.11 .2008 